\title{
Ambipolar PEDOT-Pendant Tetrachlorinated Perylene Diimide for Symmetric Supercapacitors
}

\author{
Mark B. Miltenburg, Nimrat K. Obhi, Bryony T. McAllister and Dwight S. Seferos* \\ Department of Chemistry, University of Toronto, 80 St. George Street, Toronto, Ontario M5S 3H6, Canada \\ Keywords: ambipolar polymer, supercapacitor, organic electrodes, conjugated polymers, pendant polymer
}

\begin{abstract}
Pseudocapacitive polymers offer potential for higher energy densities than electrostatic double layer capacitive materials and lower cost than pseudocapacitive metal oxides. These polymers typically demonstrate good stability when storing positive charge but poor stability when storing negative charge. The power and energy densities of these materials is also limited when the operating voltage window is restricted to positive voltages. The development of polymers capable of stable positive and negative charge storage is necessary to allow a wider voltage window and create high performance polymer supercapacitors. Here we present a PEDOTpendant tetrachlorinated perylene diimide polymer capable of storing positive and negative charge, which utilizes a donor-nodeacceptor architecture to prevent electronic interaction between positive and negative charge storing units. The polymer films show balanced charge storage and excellent stability in both positive and negative charge storage, retaining more than $80 \%$ of their capacitance over 1000 cycles. The films demonstrate moderate capacitances of $78.6 \mathrm{~F} \mathrm{~g}^{-1}$ in the positive region and $73.1 \mathrm{~F} \mathrm{~g} \mathrm{~g}^{-1}$ in the negative region at $0.5 \mathrm{~A} \mathrm{~g}^{-1}$, as well as excellent rate capabilities in positive and negative charge storage regions of $87 \%$ and $56 \%$ at $20 \mathrm{~A} \mathrm{~g}^{-1}$, respectively. The polymer film was applied as both electrodes in a symmetric type III supercapacitor device with a gel polymer electrolyte, demonstrating a wide operating potential range of $2.2 \mathrm{~V}$. These results demonstrate that the cycling stability of ambipolar polymers can be improved using a donor-node-acceptor polymer architecture with an extended $\pi$-conjugated donor unit.
\end{abstract}

\section{INTRODUCTION}

Supercapacitors are energy storage devices that are capable of delivering both high specific energy and high specific power. ${ }^{1-}$ ${ }^{3}$ High surface area carbon materials such as activated carbon, carbon nanotubes, and graphene have received significant attention as electrode materials for supercapacitors. ${ }^{4-7}$ These materials store energy through a non-Faradaic electrostatic double-layer (EDLC) mechanism, which allows very rapid charge and discharge, but limits the energy that can be stored by the accessible surface area of the electrode materials. ${ }^{8,9}$ In contrast to EDLC materials, pseudocapacitive materials such as $\mathrm{RuO}_{2}, \mathrm{MnO}_{2}$ and $\pi$-conjugated organic polymers exhibit Faradaic charge storage, with formal chemical oxidation and reduction, in addition to electrostatic charge storage. ${ }^{10-12}$ The Faradaic charge storage mechanism allows these pseudocapacitive materials to store greater amounts of energy than high surface area carbon-based materials. ${ }^{1} \pi$-conjugated polymers offer high power density, good cycle life, redox tunability by chemical modification, and potential low cost relative to the inorganic pseudocapacitive materials. ${ }^{13,14}$ However, most common $\pi$-conjugated polymer systems used in supercapacitor devices, such as poly(3,4-ethylenedioxythiophene) (PEDOT), polypyrrole, and polyaniline, are only capable of positive charging. ${ }^{3,15}$ Supercapacitors using these polymers are limited to narrow positive charging voltage windows, typically 0.8 to $1.4 \mathrm{~V}$. This voltage window restricts the amount of energy that can be stored, which is proportional to the square of the operating voltage. ${ }^{1}$ Negative charge storing polymers have been developed, however they typically suffer from poor cycling stability, and it is difficult to match charge storage across different polymers to be used in asymmetric devices. ${ }^{16}$
The optimal configuration for a polymer-based supercapacitor device would consist of two electrodes made from a single ambipolar polymer capable of stably storing both positive and negative charge, known as a Type III device. ${ }^{17,18}$ However, suitable ambipolar materials are rare, usually exhibiting relatively poor stability for negative charge storage. ${ }^{19-23}$ Recent progress on donor-acceptor $\pi$-conjugated polymers has shown that it is possible to improve negative charge storage stability by introducing electron-withdrawing groups, but this results in a corresponding reduction in positive charge storage performance. ${ }^{24-26}$ It is thus beneficial to isolate positive and negative charge storing units in order to maintain the individual performance of each. Qin et al. synthesized a donor-nodeacceptor polymer, consisting of a dicarbazole donor unit and a tetrachlorinated perylene diimide (PDI) acceptor unit. ${ }^{27}$ This donor-node-acceptor architecture decouples the performance of the positive and negative charge storage by separating the negative charge accepting PDI unit from the positive charge accepting dicarbazole through the node-like nitrogen atoms. ${ }^{28}$ Contrary to most ambipolar polymers, the donor-nodeacceptor network polymer synthesized by Qin et al. demonstrated remarkable negative region stability of $89 \%$ capacity retention after 1000 cycles, but limited positive region stability. This limited positive region stability was potentially caused by the minimal amount of possible delocalization of positive charge across the small dicarbazole donor unit, as poly(carbazole) derivatives have shown excellent cycling stabilities in previous reports..$^{29,30}$

We hypothesized that we could improve the stability of the donor-node-acceptor structure by using an extended $\pi$ conjugated polymer donor unit. Herein we report the synthesis 
of a 3,4-ethylenedioxythiophene (EDOT)-functionalized tetrachlorinated perylene diimide monomer. This monomer was electrochemically polymerized to form an ambipolar poly(EDOT)-pendant tetrachlorinated perylene diimide donornode-acceptor polymer film possessing excellent ambipolar character and remarkable stability. This synthetic strategy can be extended to vary the donor unit and solubilizing groups to optimize film properties and electrochemical behaviour. A type III polymer supercapacitor was constructed using the electropolymerized polymer films.

\section{RESULTS AND DISCUSSION}

The asymmetric EDOT-functionalized tetrachlorinated PDI was designed to provide isolated units for positive and negative charge storage. The PDI acceptor unit is able to store two electrons per unit with excellent stability. ${ }^{31,32}$ The addition of the chlorine atoms at the bay positions of the PDI unit lowers the HOMO and LUMO of the PDI unit, stabilizing the ndoped charge state and lowering the reduction potential..$^{33,34}$ The EDOT monomer unit can be electropolymerized at positive potentials without affecting the PDI unit. ${ }^{35}$ In addition, PEDOT is known to possess excellent cycling stability due to its delocalization of positive charge across extended conjugation lengths. The extended branched alkyl chain confers solubility to the monomer unit.

The node nature of the imide nitrogen atoms was examined using DFT calculations with a B3LYP functional and 6$31 \mathrm{G}(\mathrm{d})$ basis set (Figure 1). A tetrachlorinated PDI core (PDI$4 \mathrm{Cl}$ ), an EDOT monomer, and the asymmetric PDI-4Cl-EDOT monomer were modeled, with methyl groups substituted for alkyl chains for computational simplicity, and the molecular orbital energy levels were compared. The DFT calculations support the predicted node nature, as the HOMO of the PDI4Cl-EDOT is localized on the EDOT unit (Figure 1a). The LUMO is localized on the tetrachlorinated PDI core in both the PDI-4Cl and the PDI-4Cl-EDOT monomer (Figure 1b). This reinforces that the PEDOT backbone stores positive charge while the negative charge storage is localized to the tetrachlorinated perylene diimide cores. In addition, the calculated energy levels of the PDI-4Cl-EDOT monomer were compared to the component units (Table S1). The HOMO level is comparable to that of the EDOT unit, while the LUMO level is comparable to the LUMO level of the PDI-4Cl unit. This resembles traditional donor-acceptor structure polymers, where the donor contributes much of the HOMO character and the acceptor contributes much of the LUMO character. ${ }^{36}$

To carry out the synthesis, several important intermediates needed to be prepared. The PDI core was synthesized by first chlorinating the bay positions on perylene-3,4,9,10tetracarboxylic dianhydride (S1) with chlorosulfonic acid and iodine. A mixture of tri-, tetra- and penta-chlorinated derivatives was formed (S2), which was unable to be separated through chromatography or recrystallization. The crude mixture was treated with 1-butanol and 1-bromobutane, which enabled isolation of the tetrachlorinated tetraester dye (S3) by silica gel chromatography (Scheme $\mathrm{S} 1)^{37}$

The amine-functionalized EDOT unit (EDOT-MeNH $\mathrm{H}_{2}$ ) was synthesized from the chlorinated precursor (S4) by treating

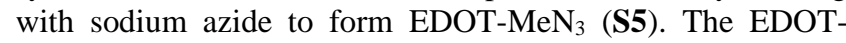
$\mathrm{MeN}_{3}$ was subsequently reduced to EDOT-MeNH (S6) using triphenylphosphine and sodium hydroxide (Scheme S2).

The asymmetric EDOT and octyl-dodecylaminefunctionalized PDI (PDI-4Cl-EDOT-O12) was synthesized from the tetrabutyl ester dye (1) (Scheme 1). The tetrabutyl ester compound was treated with $p$-toluenesulfonic acid monohydrate in refluxing heptanes to obtain the tetrachlorinated bisester dye (2). ${ }^{38}$ This reaction was extremely sensitive to temperature and reaction time, resulting in a mixture of the tetrachlorinated bisanhydride, bisester and tetrabutyl ester derivatives. The tetrabutyl ester derivative was removed by repeated washing with hexanes, and the monoanhydride was purified using Soxhlet extraction, though some of the bisanhydride compound remained. The tetrachlorinated bisester (2) was functionalized with a large branched alkyl chain to pro a)

HOMO

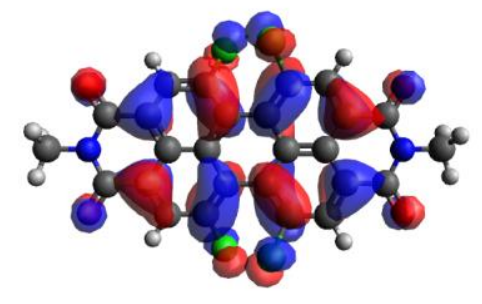

b)
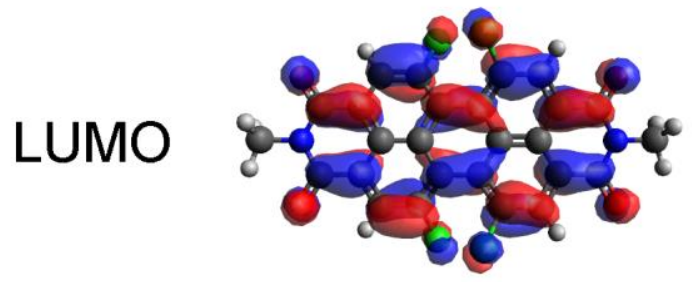

EDOT
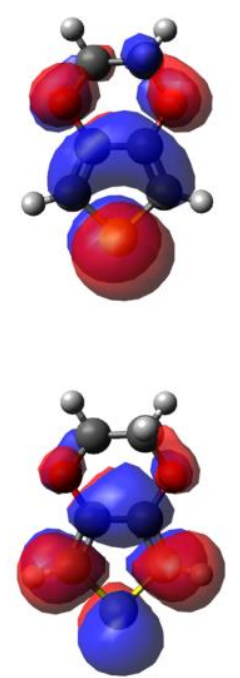

PDI-4CI-EDOT
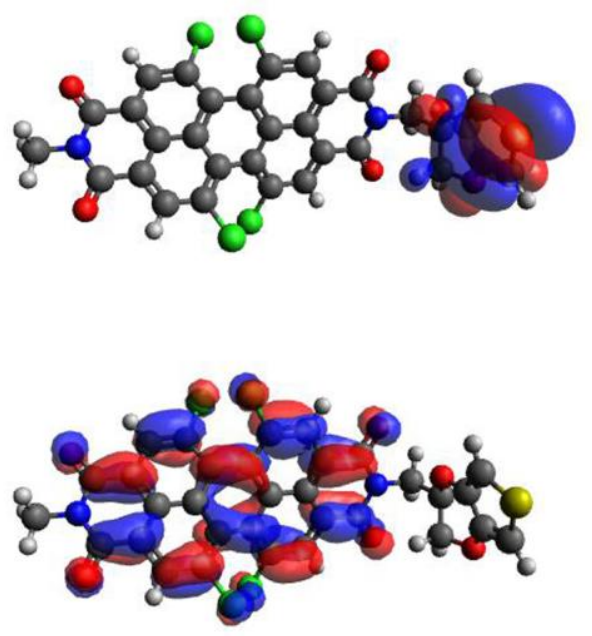

Figure 1. a) HOMO and b) LUMO diagrams of PDI-4Cl core, EDOT monomer, and asymmetric PDI-4Cl-EDOT monomer. DFT was performed using the B3LYP functional and 6-31G(d) basis set. 


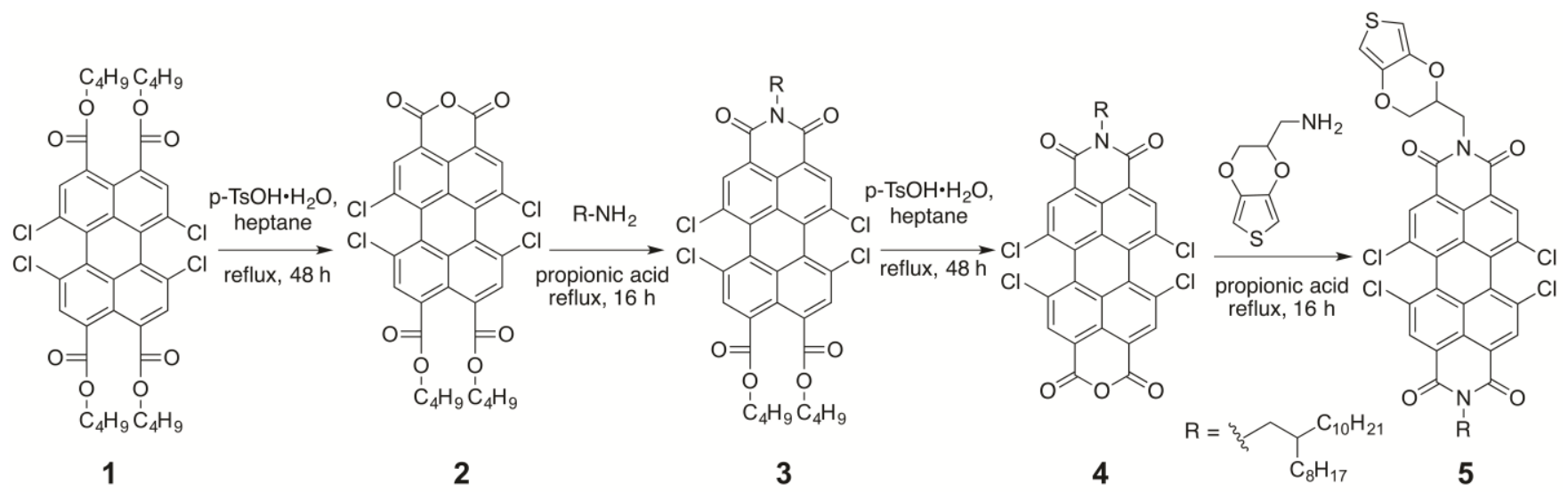

vide solubility (3). This intermediate was treated further to remove the remaining tetrabutyl esters (4) and conjugated to an EDOT unit by treating with EDOT- $\mathrm{MeNH}_{2}$ in propionic acid to form PDI-4Cl-EDOT-O12 (5). The structure of PDI4Cl-EDOT-O12 was confirmed by MALDI-TOF mass spectrometry, ${ }^{1} \mathrm{H}$ NMR spectroscopy and ${ }^{13} \mathrm{C}$ NMR spectroscopy (Supporting Information).

The PDI-4Cl-EDOT-O12 monomer was polymerized through oxidative electrochemical polymerization on a $\mathrm{Pt}$ button electrode using cyclic voltammetry $(\mathrm{CV})$. The potential was cycled from $0 \mathrm{~V}$ to $1.5 \mathrm{~V}$ relative to the ferrocene/ferrocenium $\left(\mathrm{Fc} / \mathrm{Fc}^{+}\right)$redox couple in a dichloromethane solution containing $5 \mathrm{mM}$ PDI-4Cl-EDOT-O12 and $0.1 \mathrm{M}$ tetrabutylammonium hexafluorophosphate $\left(\mathrm{TBAPF}_{6}\right)$. The current passed at the electrode surface increased with additional cycling, consistent with the formation and deposition of polymer on the electrode surface (Figure 2).

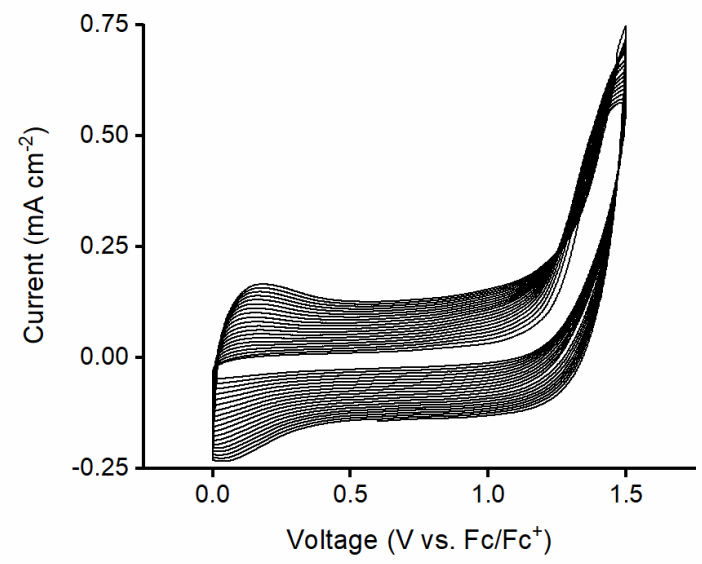

Figure 2. Cyclic voltammogram of PDI-4Cl-O12-EDOT in 0.1 M TBAPF 6 in dichloromethane at $100 \mathrm{mV} \mathrm{s}^{-1}$ (20 scans shown).

After the potential was cycled 20 times, the films were thoroughly rinsed with $0.1 \mathrm{M} \mathrm{TBAPF}_{6}$ in dichloromethane to remove any residual monomer. The mass of the polymer films was estimated by the amount of charge passed during film formation. The electrochemical characterization of the polymer films was carried out using $\mathrm{CV}$, galvanostatic charge/discharge (GCD) and electrochemical impedance spectroscopy (EIS). Cyclic voltammograms of the PEDOT/PDI polymer film were carried out separately in both the negative and positive regions to prevent charge trapping. The CVs showed balanced charge storage in both positive and negative regions, which is beneficial for a Type III supercapacitor (Figure 3). In the positive region, the film exhibited a broad delocalized redox wave indicative of a PEDOT backbone. In the negative region, there are two reversible redox processes at $-0.72 \mathrm{~V}$ and $-0.89 \mathrm{~V}$ relative to the $\mathrm{Fc} / \mathrm{Fc}^{+}$redox couple. These are associated with the reversible two electron reduction of the $\mathrm{PDI}-4 \mathrm{Cl}$ core.

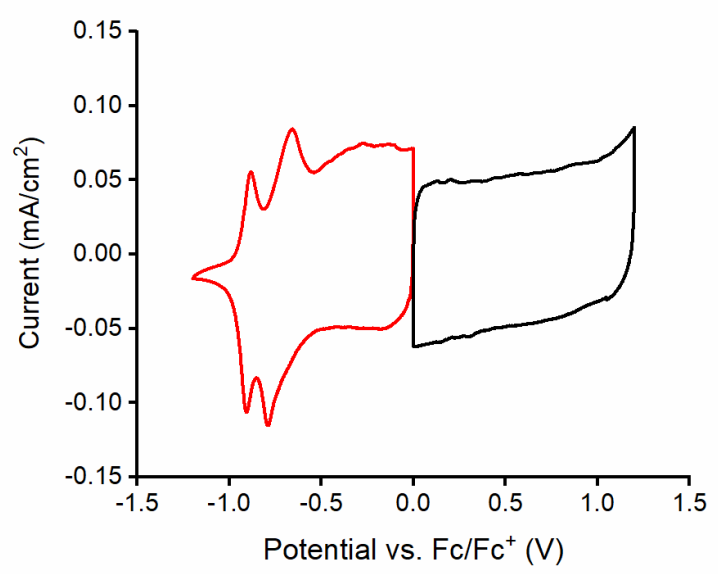

Figure 3. Positive (black) and negative (red) cyclic voltammograms of PEDOT/PDI polymer film in acetonitrile with $0.1 \mathrm{M}$ $\mathrm{TBAPF}_{6}$ at a scan rate of $10 \mathrm{mV} \mathrm{s}^{-1}$.

The redox behaviour was further examined using GCD curves (Figure 4). The positive GCD curve demonstrates expected triangular behaviour, with deviation from ideal behaviour due to the pseudocapacitance of the polymer. The small IR drop is attributed to the large, non-conductive alkyl chains causing increased resistance within the films relative to PEDOT. The negative GCD curve shows triangular behaviour with a prominent IR drop, which is attributed to poor conductivity across the PEDOT in the n-doped state, as well as the non-conductive alkyl chain mass. The capacitances of the film were calculated from GCD in both positive and negative potential ranges by integrating the area under the GCD 
curves. The positive region capacitance was $78.6 \mathrm{~F} \mathrm{~g} \mathrm{~g}^{-1}$ at 0.5 $\mathrm{A} \mathrm{g}^{-1}$, while the negative region capacitance was $73.1 \mathrm{~F} \mathrm{~g}^{-1}$ at $0.5 \mathrm{~A} \mathrm{~g} \mathrm{~g}^{-1}$, demonstrating well balanced charge storage and moderate capacitances relative to other ambipolar polymers in literature (Table S3). The moderate capacitances are attributed to two factors: the redox-inactive alkyl chain needed for monomer solubility, and potentially overestimated film masses. The large, branched alkyl chain accounts for $\sim 30 \%$ of monomer mass, but does not significantly contribute to the electrochemical properties, which decrease the capacitances relative to films without these alkyl chains. In addition, film masses were calculated based on the charge passed during the polymerization with an assumed efficiency of $100 \%$, but the coulombic efficiencies of electrochemical polymerization can be as low as $16 \%$, suggesting these capacitance values may be a significantly higher. ${ }^{39,40}$

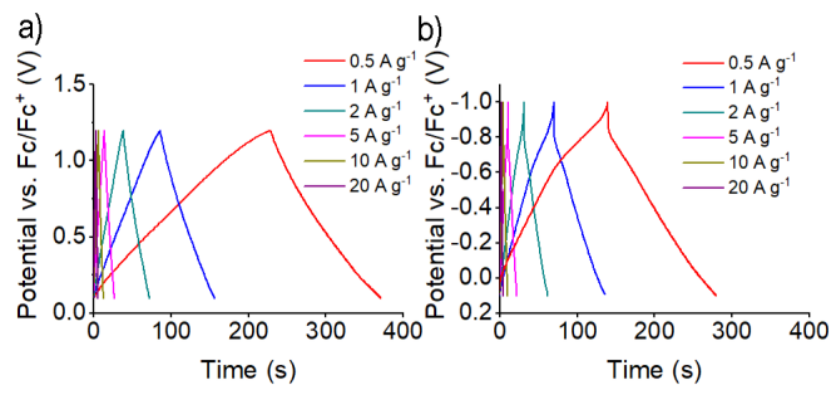

Figure 4. Galvanostatic charge-discharge of PEDOT/PDI film in a) positive and $\mathrm{b}$ ) negative regions in acetonitrile with $0.1 \mathrm{M}$ TBAPF $_{6}$

The film rate capabilities were studied at currents densities of $0.5 \mathrm{~A} \mathrm{~g}^{-1}$ to $20 \mathrm{~A} \mathrm{~g}^{-1}$. The film demonstrates excellent rate capability in the positive region, maintaining $87.4 \%$ of its capacitance at discharge current rates as high as $20 \mathrm{~A} \mathrm{~g}^{-1}$ (Figure 5a). The negative region demonstrated moderate rate capability, maintaining from $89.9 \%$ to $56.3 \%$ of capacitance across the range of increased discharge current rates. This is attributed to the increased resistance of the film in the negative region as a result of the poor conductivity through the PEDOT backbone in the $n$-doped state. ${ }^{41}$ The film was repeatedly charged and discharged in both positive and negative regions to examine capacitance retention and coulombic efficiency during cycling (Figure 5b). The film has good positive charge storage stability, retaining $79.6 \%$ of its initial capacitance after 1000 cycles at a high current rate of $10 \mathrm{~A} \mathrm{~g}$ ${ }^{1}$, with coulombic efficiencies near $95 \%$. The film also has excellent negative charge storage stability, retaining $94.7 \%$ of its initial capacitance after 1000 cycles at $5 \mathrm{~A} \mathrm{~g}^{-1}$, with coulombic efficiencies near unity. This is among the most stable positive and negative cycling of ambipolar polymers we found in literature (Figure 5c, Table S3).

The electrical conductivity and charge transport behavior of the films was probed using EIS. The films were held at six different potentials relative to $\mathrm{Fc} / \mathrm{Fc}^{+}$across a $2 \mathrm{~V}$ window during testing. Nyquist plots of the films were prepared (Figure S1a-f), and the data was fitted to an equivalent circuit (Figure S1g) to examine how the behaviour changed across the regions. The Nyquist plots show near vertical lines in the low frequency regions across multiple potentials. This suggests an accessible polymer film surface and good capacitive behaviour. In the negative regions, the resistance values vary across different potentials, which arises as a

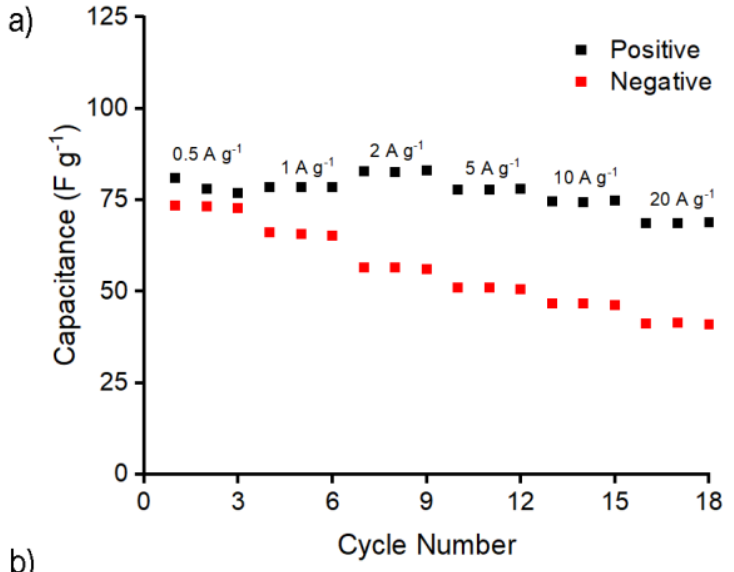

b)
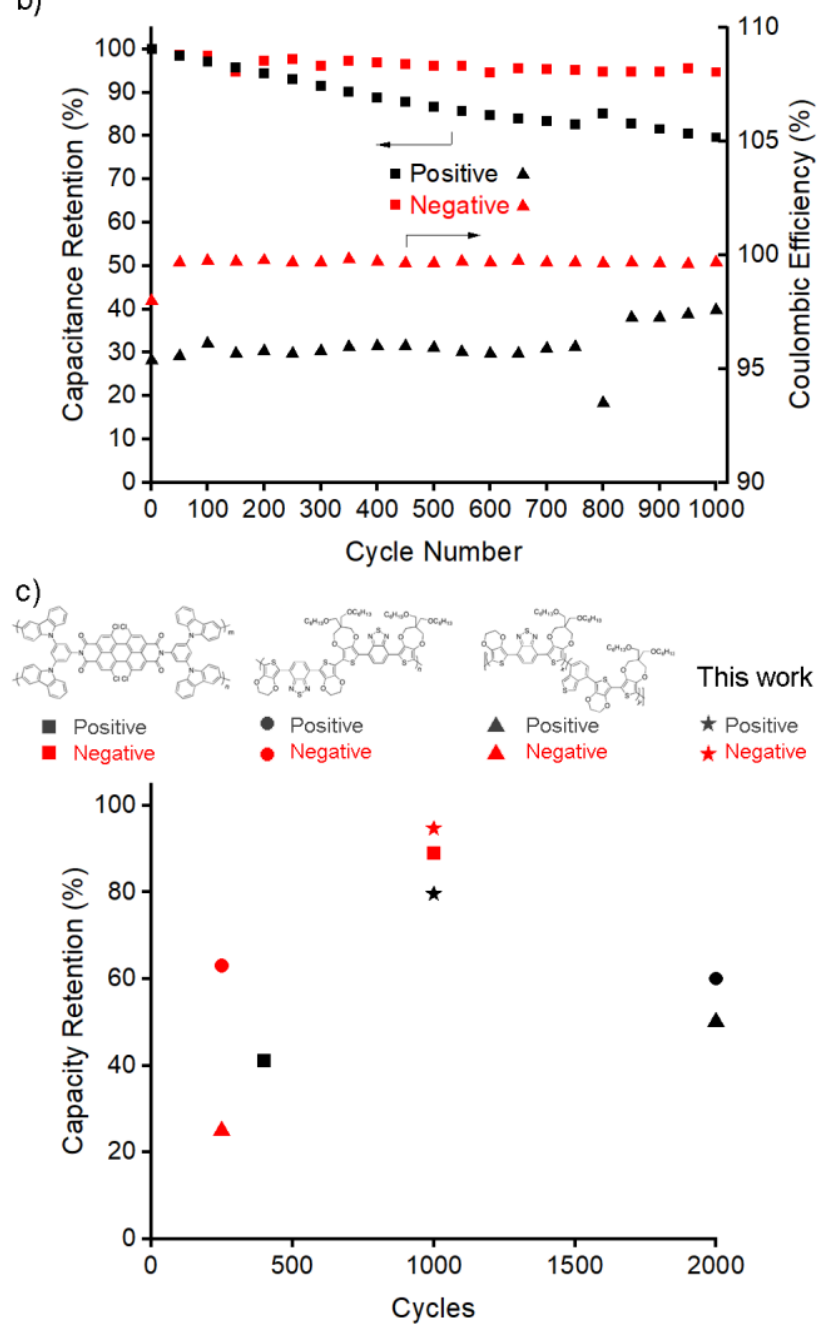

Figure 5. a) Positive (black) and negative (red) capacitances of PEDOT/PDI film at various discharge current densities. b) Capacitance retention (squares, left) and coulombic efficiencies (triangles, right) of PEDOT/PDI film in positive (black) and negative (red) regions. c) Comparison of ambipolar polymer cycling stability in positive (black) and negative (red) regions. ${ }^{24,27}$

result of the interaction of densely packed PDI units in reduced states with cations. ${ }^{42}$ In the negative region, the $\mathrm{x}$-axis of the Nyquist plot shifts to more positive values, suggesting increased resistance relative to the positive and neutral potentials. This is attributed to the difference in electron 
transport along the $\pi$-conjugated PEDOT backbone in the positive and negative potential regions. ${ }^{41}$

The potential of the films in energy storage applications, specifically as electrodes in supercapacitor devices, was examined in a Type III supercapacitor. The device was assembled using two platinum button electrodes with polymer films. Each film was deposited by electropolymerization, then the films were rinsed thoroughly with monomer-free electrolyte solution. The electrodes were charged to $0.1 \mathrm{~V}$, the midpoint of the operating range of the polymer films determined using $\mathrm{CV}$. The electrodes were coated with a poly(methyl methacrylate)/ $\mathrm{TBAPF}_{6} /$ acetonitrile gel polymer electrolyte, and placed inside a Teflon casing separated by a Celgard polypropylene separator. The devices were examined using CV (Figure 6a) and GCD (Figure 6b) across a 2.2 $\mathrm{V}$ potential range. The device produces rectangular capacitive behaviour up to about $1.5 \mathrm{~V}$, after which the rectangular $\mathrm{CV}$ profile deviated. This is attributed to charge leakage from the electrodes during device assembly. The GCD of the device showed non-ideal triangular behaviour, including a prominent IR drop, which is attributed to charge leakage from the electrodes during assembly, as well as the resistance of the polymer electrolyte and separator. The energy and power densities of the device were calculated from the discharge profiles (Figure S2). The device had a maximum energy density of $8.95 \mathrm{Wh} \mathrm{kg}^{-1}$ at $1 \mathrm{~A} \mathrm{~g}^{-1}$ and maximum power density of $76.8 \mathrm{~kW} \mathrm{~kg}^{-1}$ at $100 \mathrm{~A} \mathrm{~g}^{-1}$, demonstrating the potential of this ambipolar polymer architecture to improve the power and energy densities of supercapacitors by allowing for wider operating potential windows.

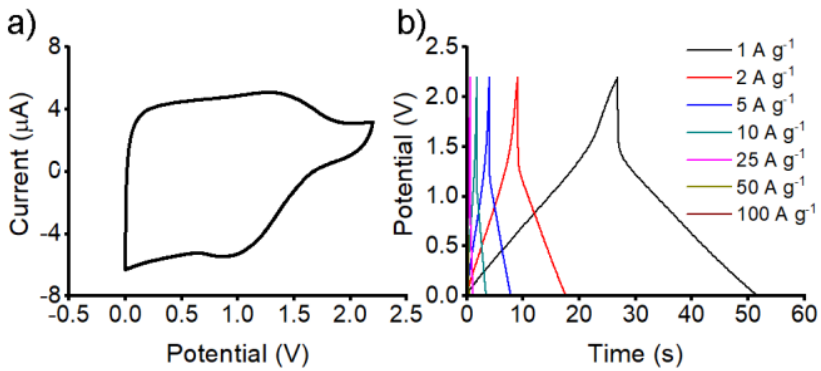

Figure 6. a) Cyclic voltammogram obtained at $100 \mathrm{mV} \mathrm{s}^{-1}$ scan rate and b) galvanostatic charge-discharge of PEDOT/PDI symmetric supercapacitor.

\section{CONCLUSIONS}

In summary, a donor-node-acceptor monomer consisting of an asymmetric tetrachlorinated perylene diimide functionalized with an EDOT monomer and a branched alkyl chain was synthesized. The monomer was electrochemically polymerized to form a PEDOT/PDI polymer film. Cyclic voltammetry of the polymer film reveals balanced positive and negative charge storage with a wide operating potential window of $2.2 \mathrm{~V}$. Galvanostatic charge-discharge experiments show that the polymer films has moderate capacitances of 78.6 $\mathrm{F} \mathrm{g}^{-1}$ (positive region) and $73.1 \mathrm{~F} \mathrm{~g}^{-1}$ (negative region) and excellent rate capabilities in the positive region, retaining $87.4 \%$ capacitance at $20 \mathrm{~A} \mathrm{~g}^{-1}$, with moderate rate capabilities in the negative region. It is expected that modifying the side chain used to confer solubility to the monomer could improve the performance of this polymer further. Cycling experiments show excellent stability in both regions, retaining $79.6 \%$ capacitance after 1000 cycles in the positive re- gion and $94.7 \%$ in the negative region at high current densities. These are some of the most stable ambipolar polymer electrodes to date, making them ideal for application in energy storage devices such as supercapacitors. The polymer affords a Type III supercapacitor using a gel polymer electrolyte, operating over a wide voltage window of $2.2 \mathrm{~V}$, and demonstrating high maximum energy and power densities of $8.95 \mathrm{Wh} \mathrm{kg}^{-1}$ and $76.8 \mathrm{~kW} \mathrm{~kg}^{-1}$ respectively.

\section{ASSOCIATED CONTENT}

\section{Supporting Information}

Supporting information contains an experimental section, supplementary figures, schemes and tables, and a complete Gaussian 09 citation.

\section{AUTHOR INFORMATION}

\section{Corresponding Author}

*E-mail: dseferos@chem.utoronto.ca

\section{Author Contributions}

All authors have given approval to the final version of the manuscript.

\section{Funding Sources}

This work was supported by the Natural Sciences and Engineering Research Council of Canada (NSERC), the Canadian Foundation for Innovation, and the Ontario Research Fund.

\section{ACKNOWLEDGMENT}

The authors would like to acknowledge Dr. George McKeown for writing scripts used in analysis of GCD data. This research was enabled in part by support provided by Compute Ontario (www.computeontario.ca) and Compute Canada (www.computecanada.ca).

\section{ABBREVIATIONS}

B3LYP, Becke, 3-parameter, Lee-Yang-Parr; CV, cyclic voltammetry; DFT, density functional theory; EDLC, electric double layer capacitance; EDOT, 3,4-ethylenedioxythiophene; EIS, electrochemical impedance spectroscopy; $\mathrm{Fc} / \mathrm{Fc}^{+}$, ferrocene/ferrocenium; GCD, galvanostatic charge-discharge; HOMO, highest occupied molecular orbital; LUMO, lowest unoccupied molecular orbital; MALDI-TOF, matrix-assisted laser desorption/ionization-time of flight; NMR, nuclear magnetic resonance, PDI, perylene diimide; PEDOT, poly $(3,4-$ ethylenedioxythiophene); $\mathrm{TBAPF}_{6}$, tetrabutylammonium hexafluorophosphate.

\section{REFERENCES}

(1) Conway, B. E. Electrochemical Supercapacitors; Kluwer Academic/Plenum Publishers, New York, 1999.

(2) Simon, P.; Gogotsi, Y.; Dunn, B. Where Do Batteries End and Supercapacitors Begin? Science 2014, 343 (6176), 12101211.

(3) Schon, T. B.; McAllister, B. T.; Li, P.-F.; Seferos, D. S. The Rise of Organic Electrode Materials for Energy Storage. Chem. Soc. Rev. 2016, 45, 6345-6404.

(4) Zhang, L. L.; Zhao, X. S. Carbon-Based Materials as Supercapacitor Electrodes. Chem. Soc. Rev. 2009, 38, 2520-2531.

(5) Wang, Y.; Shi, Z.; Huang, Y.; Ma, Y.; Wang, C.; Chen, M.; Chen, Y. Supercapacitor Devices Based on Graphene Materials. J. Phys. Chem. C 2009, 113 (30), 13103-13107. 
(6) Borenstein, A.; Hanna, O.; Attias, R.; Luski, S.; Brousse, T.; Aurbach, D. Carbon-Based Composite Materials for Supercapacitor Electrodes: a Review. J. Mater. Chem. A 2017, 5, 12653 12672 .

(7) Wang, Q.; Yan, J.; Fan, Z. Carbon Materials for High Volumetric Performance Supercapacitors: Design, Progress, Challenges and Opportunities. Energy Environ. Sci. 2016, 9, 729-762.

(8) Béguin, F.; Presser, V.; Balducci, A.; Frackowiak, E. Carbons and Electrolytes for Advanced Supercapacitors. Adv. Mater. 2014, 26 (14), 2219-2251.

(9) Electrochemical Double-Layer Capacitors (EDLC). In Supercapacitors Based on Carbon or Pseudocapacitive Materials; John Wiley \& Sons, Ltd, 2017; pp 1-25.

(10) Pseudocapacitive Materials. In Supercapacitors Based on Carbon or Pseudocapacitive Materials; John Wiley \& Sons, Ltd, 2017; pp 39-55.

(11) Augustyn, V.; Simon, P.; Dunn, B. Pseudocapacitive Oxide Materials for High-Rate Electrochemical Energy Storage. Energy \& Environmental Science 2014, 7 (5), 1597-1614.

(12) Brousse, T.; Bélanger, D.; Long, J. W. To Be or Not to Be Pseudocapacitive? J. Electrochem. Soc. 2015, 162 (5), A5185A5189.

(13) Conway, B. E.; Pell, W. G. Double-Layer and Pseudocapacitance Types of Electrochemical Capacitors and Their Applications to the Development of Hybrid Devices. J. Solid State Electr. 2003, 7 (9), 637-644.

(14) Fong, K. D.; Wang, T.; Smoukov, S. K. Multidimensional Performance Optimization of Conducting Polymer-Based Supercapacitor Electrodes. Sustain. Energy Fuels 2017, 1, 1857-1874.

(15) Meng, Q.; Cai, K.; Chen, Y.; Chen, L. Research Progress on Conducting Polymer Based Supercapacitor Electrode Materials. Nano Energy 2017, 36, 268-285.

(16) Bryan, A. M.; Santino, L. M.; Lu, Y.; Acharya, S.; D’Arcy, J. M. Conducting Polymers for Pseudocapacitive Energy Storage. Chem. Mater. 2016, 28 (17), 5989-5998.

(17) Sharma, S.; Soni, R.; Kurungot, S.; Asha, S. K. Rylene Diimide-Based Alternate and Random Copolymers for Flexible Supercapacitor Electrode Materials with Exceptional Stability and High Power Density. J. Phys. Chem. C 2019, 123 (4), 2084-2093.

(18) Wang, K.; Huang, L.; Eedugurala, N.; Zhang, S.; Sabuj, M. A.; Rai, N.; Gu, X.; Azoulay, J. D.; Ng, T. N. Wide Potential Window Supercapacitors Using Open-Shell Donor-Acceptor Conjugated Polymers with Stable N-Doped States. Adv. Energy Mater. 2019, 9 (47), 1902806-1902808.

(19) Estrada, L. A.; Liu, D. Y.; Salazar, D. H.; Dyer, A. L.; Reynolds, J. R. Poly[Bis-EDOT-Isoindigo]: an Electroactive Polymer Applied to Electrochemical Supercapacitors. Macromolecules 2012, 45 (20), 8211-8220.

(20) DiCarmine, P. M.; Schon, T. B.; McCormick, T. M.; Klein, P. P.; Seferos, D. S. Donor-Acceptor Polymers for Electrochemical Supercapacitors: Synthesis, Testing, and Theory. J. Phys. Chem. C 2014, 118 (16), 8295-8307.

(21) Sun, Y.; Zhao, X.; Zhu, G.; Li, M.; Zhang, X.; Yang, H.; Lin, B. Twisted Ladder-Like Donor-Acceptor Polymers as Electrode Materials for Flexible Electrochromic Supercapacitors. Electrochim. Acta 2020, 333, 135495.

(22) Fusalba, F.; Ho, H. A.; Breau, L.; Bélanger, D. Poly(Cyano-Substituted Diheteroareneethylene) as Active Electrode Material for Electrochemical Supercapacitors. Chem. Mater. 2000, 12 (9), 2581-2589.

(23) Ferraris, J. P.; Eissa, M. M.; Brotherston, I. D.; Loveday, D. C. Performance Evaluation of Poly 3-(Phenylthiophene) Derivatives as Active Materials for Electrochemical Capacitor Applications. Chem. Mater. 1998, 10 (11), 3528-3535.

(24) Wang, Y.; Li, W.; Guo, Y.; Cao, J.; Murtaza, I.; Syed, A. S.; He, Y.; Meng, H. Recombination Strategy for Processable Ambipolar Electroactive Polymers in Pseudocapacitors. Macromolecules 2018, 51 (14), 5258-5266.

(25) Levi, M. D.; Aurbach, D. A Short Review on the Strategy Towards Development of П-Conjugated Polymers with Highly
Reversible P- and N-Doping. J. Power Sources 2008, 180 (2), 902 908 .

(26) Pron, A.; Gawrys, P.; Zagorska, M.; Djurado, D.; Demadrille, R. Electroactive Materials for Organic Electronics: Preparation Strategies, Structural Aspects and Characterization Techniques. Chem. Soc. Rev. 2010, 39, 2577-2632.

(27) Qin, L.; Ma, W.; Hanif, M.; Jiang, J.; Xie, Z.; Ma, Y. Donor-Node-Acceptor Polymer with Excellent N-Doped State for High-Performance Ambipolar Flexible Supercapacitors. Macromolecules 2017, 50 (9), 3565-3572.

(28) Würthner, F. Perylene Bisimide Dyes as Versatile Building Blocks for Functional Supramolecular Architectures. Chem. Commun. 2004, 1564-1579.

(29) Ates, M.; Özten, E. The Comparison of Capacitor Behaviors of Polymethylcarbazole and Polymethylcarbazole/Graphene. $J$. Alloys Compd. 2017, 714, 433-442.

(30) Wang, H.; Cheng, Z.; Liao, Y.; Li, J.; Weber, J.; Thomas, A.; Faul, C. F. J. Conjugated Microporous Polycarbazole Networks as Precursors for Nitrogen-Enriched Microporous Carbons for $\mathrm{CO} 2$ Storage and Electrochemical Capacitors. Chem. Mater. 2017, 29 (11), 4885-4893.

(31) Song, Z.; Zhan, H.; Zhou, Y. Polyimides: Promising Energy-Storage Materials. Angew. Chem. Int. Ed. 2010, 49 (45), 8444 8448 .

(32) Huang, C.; Barlow, S.; Marder, S. R. Perylene-3,4,9,10Tetracarboxylic Acid Diimides: Synthesis, Physical Properties, and Use in Organic Electronics. J. Org. Chem. 2011, 76 (8), 2386-2407.

(33) Chen, Z.; Debije, M. G.; Debaerdemaeker, T.; Osswald, P.; Würthner, F. Tetrachloro-Substituted Perylene Bisimide Dyes as Promising N-Type Organic Semiconductors: Studies on Structural, Electrochemical and Charge Transport Properties. ChemPhysChem 2004, 5 (1), 137-140.

(34) Delgado, M. C. R.; Kim, E.-G.; Filho, D. A. D. S.; Bredas, J.-L. Tuning the Charge-Transport Parameters of Perylene Diimide Single Crystals via End and/or Core Functionalization: a Density Functional Theory Investigation. J. Am. Chem. Soc. 2010, 132 (10), 3375-3387.

(35) Segura, J. L.; Gómez, R.; Reinold, E.; Bäuerle, P. Synthesis and Electropolymerization of a PerylenebisimideFunctionalized 3,4-Ethylenedioxythiophene (EDOT) Derivative. Org. Lett. 2005, 7 (12), 2345-2348.

(36) Holliday, S.; Li, Y.; Luscombe, C. K. Recent Advances in High Performance Donor-Acceptor Polymers for Organic Photovoltaics. Prog. Polym. Sci. 2017, 70, 34-51.

(37) Dubey, R. K.; Westerveld, N.; Grozema, F. C.; Sudhölter, E. J. R.; Jager, W. F. Facile Synthesis of Pure 1,6,7,12Tetrachloroperylene-3,4,9,10-Tetracarboxy Bisanhydride and Bisimide. Org. Lett. 2015, 17 (8), 1882-1885.

(38) Dubey, R. K.; Westerveld, N.; Sudhölter, E. J. R.; Grozema, F. C.; Jager, W. F. Novel Derivatives of 1,6,7,12Tetrachloroperylene-3,4,9,10-Tetracarboxylic Acid: Synthesis, Electrochemical and Optical Properties. Org. Chem. Front. 2016, 3, 1481-1492.

(39) Szkurlat, A.; Palys, B.; Mieczkowski, J.; Skompska, M. Electrosynthesis and Spectroelectrochemical Characterization of Poly(3,4-Dimethoxy-Thiophene), Poly(3,4-Dipropyloxythiophene) and Poly(3,4-Dioctyloxythiophene) Films. Electrochim. Acta 2003, 48 (24), 3665-3676.

(40) Zotti, G.; Zecchin, S.; Schiavon, G.; Groenendaal, L. B. Conductive and Magnetic Properties of 3,4-Dimethoxy- and 3,4Ethylenedioxy-Capped Polypyrrole and Polythiophene. Chem. Mater. 2000, 12 (10), 2996-3005.

(41) Ahonen, H. J.; Lukkari, J.; Kankare, J. N- and P-Doped Poly(3,4-Ethylenedioxythiophene): Two Electronically Conducting States of the Polymer. Macromolecules 2000, 33 (18), 6787-6793.

(42) DeBlase, C. R.; Hernández-Burgos, K.; Rotter, J. M.; Fortman, D. J.; S Abreu, dos, D.; Timm, R. A.; Diógenes, I. C. N.; Kubota, L. T.; Abruña, H. D.; Dichtel, W. R. Cation-Dependent Stabilization of Electrogenerated Naphthalene Diimide Dianions in Porous Polymer Thin Films and Their Application to Electrical 
Energy Storage. Angew. Chem. Int. Ed. 2015, 54 (45), 1322513229. 

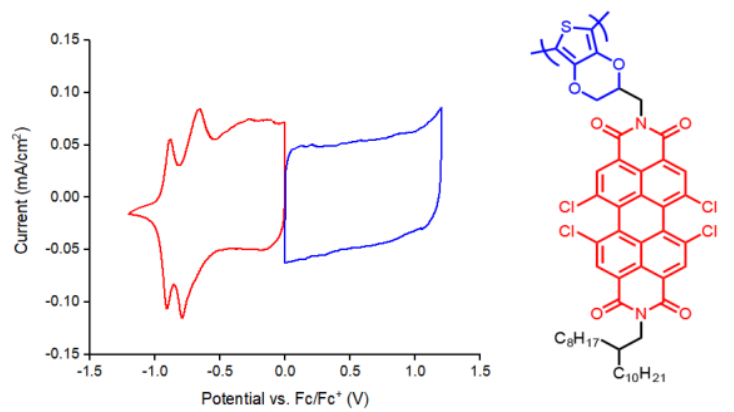